\title{
Macrolides with Promotive Activity of Monocyte to Macrophage Differentiation
}

\author{
Kiminari Yoshida, Toshiaki Sunazuka, Kenichiro Nagai, Akihiro Sugawara, Achim Cho, \\ Tohru Nagamitsu, Yoshihiro Harigaya, Kazuhiko Otoguro, Kiyoko S. Akagawa, \\ Satoshi Ōmura
}

Received: November 11, 2004 / Accepted: December 6, 2004

(C) Japan Antibiotics Research Association

\begin{abstract}
We have been interested in the immunomodulatory effect, to promote differentiation of the human monocytic cell line THP-1 to macrophages of EMA. We chemically modified EM-A in order to obtain derivatives with stronger promoting activity of monocyte to macrophage differentiation and no antibacterial activity. Most of the EM701 derivatives produced, all 12-membred macrolides, were remarkably active and were free of antibacterial activity. Among them, the most potent derivative was EM703, which showed very weak gastrointestinal motor-stimulating activity. EM703 may be useful tool to study the mechanisms of action of macrophage differentiation, and may be lead candidate for the development of new therapeutic drugs for chronic airway disease.
\end{abstract}

Keywords macrolide, 12-membered macrolide, novel actions, immunomodulatory effect, anti-inflammatory effect, macrophage differentiation

Macrolide antibiotics are widely used as antimicrobial agents. Previously, we discovered strong gastrointestinal motor-stimulating (GMS) activity by erythromycin A (EM-
A) and its non-antimicrobial derivatives [1, 2], and the generic name 'motilide' was proposed for a series of macrolides having motilin-agonistic activity [3 6].

Recently, EM-A and other macrolide antibiotics have been shown to be efficacious against chronic inflammatory airway disease, in addition to having antibacterial activity. The prognosis for diffuse panbronchiolitis (DPB), an incurable chronic inflammatory airway disease, improved significantly in two studies upon treatment with long term and low doses of such macrolide antibiotics $[7,8]$. Such therapeutic efficacy is thought to be caused by either antiinflammatory or immunomodulatory activity of macrolide antibiotics.

EM-A and azithromycin (AZM), one of the 15membered ring macrolide antibiotics, have been shown to have inhibitory activity against the inflammatory functions of neutrophils, in vitro [9]. The two antibiotics also have a clinical effect in the treatment of DPB. Furthermore, EM-A has exhibited a prophylactic effect on lung injury in vivo in a bleomycin-induced acute lung injury rat model [10].

We previously clarified the suppressive effect of interleukin (IL)-8 release in a human bronchial epithelial cell line by EM-A, clarithromycin (CAM) and EMderivatives [11]. We also examined the anti-inflammatory
S. Ōmura (Corresponding author), K. Yoshida, T. Sunazuka, K. Nagai, A. Sugawara, A. Cho, T. Nagamitsu, Y. Harigaya, K. Otoguro: Kitasato Institute for Life Sciences and Graduate School of Infection Control Sciences, and School of Pharmaceutical Sciences, Kitasato University and The Kitasato Institute, 5-9-1 Shirokane, Minato-ku, Tokyo 108-8641, Japan, Email: omura-s@kitasato.or.jp
K. S. Akagawa: National Institute of Infectious Disease, 1 Toyama, Shinjuku-ku, Tokyo 162-8640, Japan 


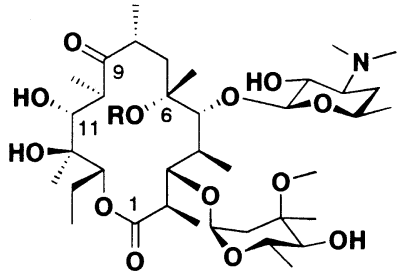

Erythromycin (EM-A) $\mathbf{R}=\mathbf{H}$ Clarithromycin (CAM) $R=M e$
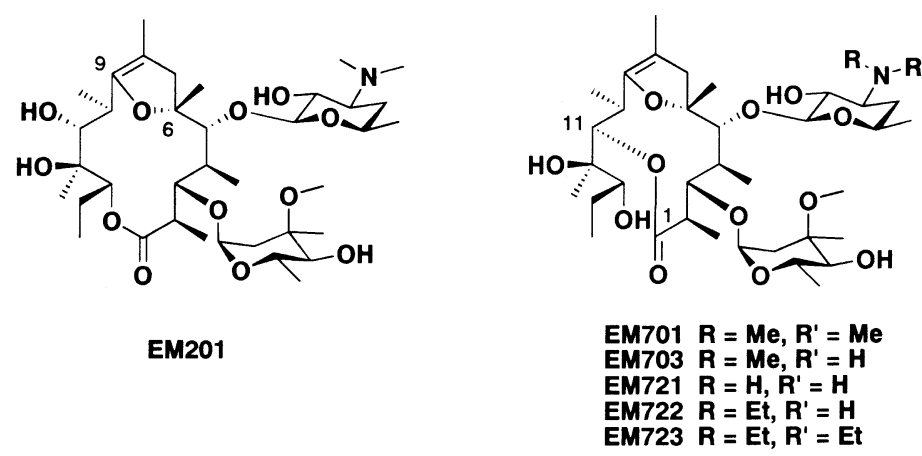

Fig. 1 Structures of erythromycin A, its derivatives and clarithromycin.

Table 1 Biological activities of EM-A, CAM and EM derivatives

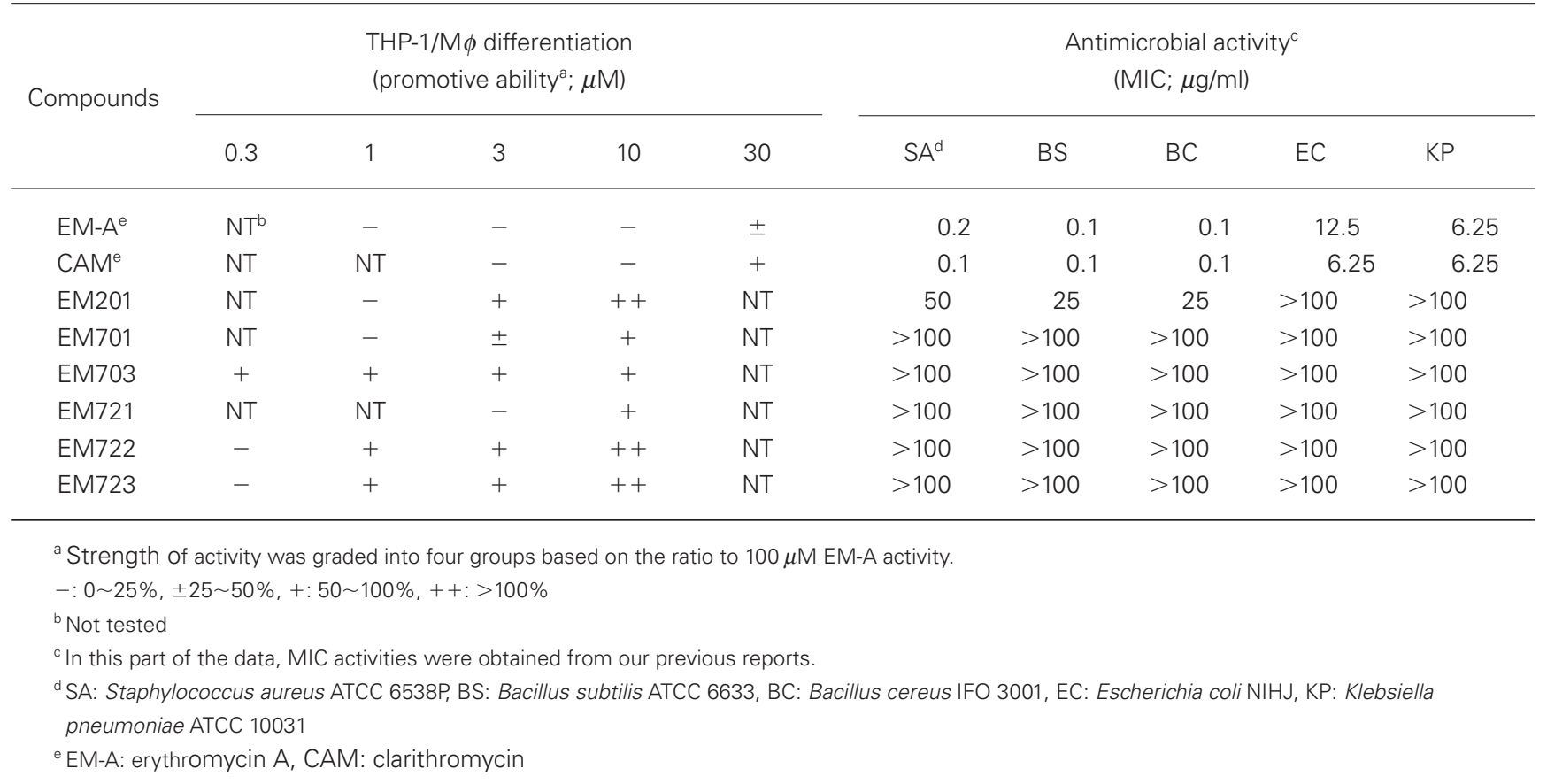

effects of EM-A, CAM, roxithromycin (RXM) and nonantimicrobial EM-derivatives by examining their inhibitory activity against rat leucocyte chemotaxis [12]. EM-A, CAM and RXM exhibited only weak chemotaxis inhibitory activity, while the non-antimicrobial EM-derivatives showed remarkable activity. Thus, the structural factors involved in antibacterial and anti-inflammatory activity are distinct.

On the other hand, we also reported on the immunomodulatory effect, to promote differentiation of the human monocytic cell line THP-1 to macrophages, of EMA, CAM, RXM and EM-derivatives with no or weak antimicrobial activity in vitro $[13,14]$.

We believe that non-antibacterial EM derivatives could have various immunopharmacological activities, and that analysis of the mechanisms of action involved should be useful for development of new therapeutic drugs for chronic airway disease.

On the basis of the above findings, we chemically modified EM-A in order to obtain derivatives with stronger promoting activity of monocyte to macrophage differentiation and no antibacterial activity.

The promotive activities were determined by modifying the method of Keicho et al. (1994) [13]. THP-1 cell line, derived from a patient with monocytic leukemia, was supplied by the Japanese Cancer Research Resources Bank (Tokyo, Japan. Now the Japan Health Sciences Foundation).

THP- 1 cells $\left(1 \times 10^{5}\right.$ per well in $\left.0.5 \mathrm{ml}\right)$ were seeded into 48-well tissue culture microplates (IWAKI, Japan) and cultured in the presence of phorbol myristate acetate (PMA; $2 \mathrm{ng} / \mathrm{ml})$, each macrolide compound $(0.3 \sim 30 \mu \mathrm{M})$ alone, or 
with macrolide compound and PMA, for 4 days at $37^{\circ} \mathrm{C}$ under $5 \% \mathrm{CO}_{2}$ humidified air. The number and viability of adherent cells were measured by colorimetric determination of MTT ((3-(4,5-dimethylthiazol-2-yl)-2,5-diphenyl) tetrazolium bromide) at $550 \mathrm{~nm}$.

Of the erythromycin derivatives, 8,9-anhydroerythromycin A 6,9-hemiketal (EM201), obtained by mild acid treatment of EM-A showed a promotive effect on THP-1 cell differentiation at $10 \mu \mathrm{M}$, and weak antimicrobial activity [14]. Next, EM201 was treated with $\mathrm{K}_{2} \mathrm{CO}_{3}$ in $\mathrm{MeOH}$ to afford the 12-membered macrolide, pseudoerythromycin A (EM701) [15]. Furthermore, de- $N$ methyl (EM703), and bis-de- $N$-methyl (EM721) derivatives were obtained by treating EM701 with $\mathrm{I}_{2} / \mathrm{NaOAc}$, and $\mathrm{I}_{2} / \mathrm{NaOMe}$ [16]. $N$-Ethyl (EM722), and bis- $N$-ethyl (EM723) derivatives were obtained by treating EM721 with EtI/diisopropylethylamine.

Most of the EM701 derivatives, 12-membred macrolides listed in Table 1, were remarkably active and were free of antibacterial activity. Among them, the most potent derivative was EM703, which showed very weak gastrointestinal motor-stimulating activity.

Furthermore, the preliminary results indicated that EM703 exhibited a prophylactic effect on lung injury in vivo against a bleomycin-induced acute lung injury rat model, similar to EM-A.

In conclusion, the EM701 derivatives illustrated here may alone be useful tools to study the mechanisms of action of, and may be lead candidates for the development of, new therapeutic drugs for chronic airway disease.

Acknowledgments This work was supported by a Grant of the 21st Century COE Program, Ministry of Education, Culture, Sports, Science and Technology (MEXT), The Japan Science and Technology Corporation (JST), a Grant-in-Aid for Scientific Research from the Ministry of Education, Science, Sports and Culture, Japan, and the Japan Keirin Association.

\section{References}

1. Ōmura S, Tsuzuki K, Sunazuka T, Toyoda H, Takahashi I, Itoh Z. Gastrointestinal motor-stimulating activity of macrolide antibiotics and the structure-activity relationship. J Antibiot 38: 1631-1632 (1985)

2. Ōmura S, Tsuzuki K, Sunazuka T, Marui S, Toyoda H, Inatomi $\mathrm{N}$, Itoh $\mathrm{Z}$. Macrolides with gastrointestinal motor stimulating activity. J Med Chem 30: 1941-1943 (1987)

3. Tsuzuki K, Sunazuka T, Marui S, Toyoda H, Ōmura S. Motilides, macrolides with gastrointestinal motor stimulating activity. I. $O$-Substituted and tertiary $N$ substituted derivatives of 8,9-anhydroerythromycin A 6,9hemiacetal. Chem Pharm Bull 37: 2687-2700 (1989)

4. Sunazuka T, Tsuzuki K, Marui S, Toyoda H, Ōmura S, Inatomi N, Itoh $\mathrm{Z}$. Motilides, macrolides with gastrointestinal motor stimulating activity. II. Quaternary $N$ substituted derivatives of 8,9-anhydroerythromycin A 6,9hemiacetal and 9,9-dihydroerythromycin A 6,9-epoxide. Chem Pharm Bull 37: 2701-2709 (1989)

5. Kondo Y, Torii K, Ōmura S, Itoh Z. Erythromycin and its derivatives with motilin-like biological activities inhibit the specific binding of ${ }^{125}$ I-motilin to duodenal muscle. Biochem Biophys Res Commun 150: 877-882 (1988)

6. Ōmura S, Kondo Y, Itoh Z. Motilide, motilin-like macrolide. In Motilin, Ed., Itoh Z. Academic Press, New York, pp. 245-256 (1990)

7. Kudoh S, Uetake T, Hagiwara K, Hirayama M, Lh H, Kimura H, Sugiyama Y. Clinical effect of low dose long term erythromycin chemotherapy on diffuse panbronchiolitis. Jap J Thorac Dis 25: 632-642 (1987)

8. Kadota J, Sakito O, Kohno S, Sawa H, Mukae H, Oda H, Kawakami K, Fukushima K, Hiratani K, Hara K. A mechanism of erythromycin treatment in patients with diffuse panbronchiolitis. Am Rev Respir Dis 147: 153-159 (1993)

9. Sugihara E. Effect of macrolide antibiotics on neutrophil function in human peripheral blood. Kansenshogaku Zasshi 71: 329-336 (1997) in Japanese

10. Azuma A, Furuta T, Enomoto T, Hashimoto Y, Uematsu K, Nukariya N, Murata A, Kudoh S. Preventive effect of erythromycin on experimental bleomycin-induced acute lung injury in rats. Thorax 53: 186-189 (1998)

11. Sunazuka T, Takizawa H, Desaki M, Suzuki K, Obata R, Otoguro K, Ōmura S. Effects of erythromycin and its derivatives on interleukin- 8 release by human bronchial epithelial cell line BEAS-2B Cells. J Antibiot 52: 71-74 (1999)

12. Oohori M, Otoguro K, Sunazuka T, Suzuki K, Iwai Y, Ömura S. Effect of 14-membered ring macrolide compounds on rat leucocytes chemotaxis and the structure-activity relationships. J Antibiot 53: 1219-1222 (2000)

13. Keicho N, Kudoh S, Yotsumoto H, Akagawa KS. Erythromycin promotes monocyte to macrophage differentiation. J Antibiot 47: 80-89 (1994)

14. Sunazuka T, Oohori M, Otoguro K, Suzuki K, Iwai Y, Akagawa KS, Ōmura S. Effect of 14-membered macrolide compounds on monocyte to macrophage differentiation. J Antibiot 56: 721-724 (2003)

15. Kirst HA, Wind JA, Paschal JW. Synthesis of ringcontracted derivatives of erythromycin. J Org Chem 52: 4359-4362 (1987)

16. Freiberg LA. Japan Kokai Patent 47-9129 (1972) 